\title{
CERTAIN RESULTS OF REAL HYPERSURFACES IN A COMPLEX SPACE FORM
}

\author{
AMALENDU GHOSH \\ Department of Mathematics, Krishnagar Government College, Krishnagar: 741101, W.B., India \\ e-mail: aghosh_70@yahoo.com
}

(Recieved 4 August 2010; revised 17 December 2010; accepted 11 April 2011; first published online 2 August 2011)

\begin{abstract}
First, we classify a real hypersurface of a non-flat complex space form with (i) semi-parallel $T\left(=£_{\xi} g\right.$ ), and (ii) recurrent $T$. Next, we characterise a real hypersurface admitting the generalised $\eta$-Ricci soliton in a non-flat complex space form.
\end{abstract}

2010 Mathematics Subject Classification. 53B20, 53C15, 53C25.

1. Introduction. A complex $n$-dimensional Kaehler manifold of constant holomorphic sectional curvature $c$ is called a complex space form and is denoted by $\bar{M}^{n}(c)$. A complete and simply connected complex space form is a complex Euclidean space $C^{n}$, if $c=0$, a complex projective space $P_{n}(C)$, if $c>0$ or a complex hyperbolic space $H_{n}(C)$, if $c<0$. Takagi $[17,18]$ first characterised all homogeneous real hypersurfaces in $P_{n}(C)$ into six model spaces $A_{1}, A_{2}, B, C, D$ and $E$. Thereafter, Cecil and Ryan [2] (see also [9]) studied extensively that when the structure vector field $\xi$ is principal and showed that they are realised as the tubes over certain submanifolds in $P_{n}(C)$ by using its focal map. On the other hand, Berndt [1] classified all homogeneous real hypersurfaces in $H_{n}(C)$ with $\xi$ as principal vector and divided into four model space $A_{0}, A_{1}, A_{2}$ and $B$. Let $M$ be a real hypersurface of a non-flat complex space form. Then $M$ has an almost contact metric structure $(\varphi, \xi, \eta, g)$ induced from the complex structure $J$. Many differential geometers studied real hypersurfaces of a complex space form under various conditions on the Ricci tensor, the shape operator $A$ (in the direction of the unit normal of $M$ ), curvature tensor etc. For a real hypersurface of a complex space form, we now define the tensor $T$ by

$$
g(T X, Y)=\left(£_{\xi} g\right)(X, Y)=g((\varphi A-A \varphi) X, Y),
$$

for all vector fields $X, Y$ tangent to $M$. A typical characterisation for a real hypersurface $M$ of type $A$ in a complex space form $\bar{M}^{n}(c)$ was given under the condition $g(T X, Y)=$ 0 , for any tangent vector fields $X$ and $Y$ on $M$. Under this condition Okumura [15], for $c>0$, and Montiel-Romero [13], for $c<0$ proved the following:

THEOREM A. Let $M^{2 n-1}$ be a real hypersurface in a non-flat complex space form. If $M$ satisfies $A \varphi=\varphi A$, then $M$ is locally congruent to real hypersurface of type $A$.

Let $M$ be a real hypersurface of type $A$ in $\bar{M}^{n}(c)$. Then it follows from Theorem A that M naturally satisfies $\nabla_{X} T=0$. Thus, as a generalisation of Okumura's condition $g(T X, Y)=0$, for any tangent vector fields $X$ and $Y$ on $M$, here we consider the real 
hypersurfaces $M$ of a non-flat complex space form $\bar{M}^{n}(c)$ with semi-parallel tensor $T$ (i.e. $R \cdot T=0$, where $R$ is the curvature tensor of $M$ ) and prove that such hypersurface is the Hopf hypersurface and also locally congruent to one of type $A$ in $P_{n}(C)$ or $H_{n}(C)$. We also consider a real hypersurface of a non-flat complex space form with recurrent $T$ and prove that such hypersurface is locally congruent to one of type $A$ in $P_{n}(C)$ or $H_{n}(C)$. We discuss these issues in Section 3.

It is well known [5] that there are no real hypersurfaces with parallel Ricci tensor in a non-flat complex space form $\bar{M}^{n}(c)$ when $n \geq 3$. This is also true for $n=2$ as was pointed out by Kim [7]. Since the Einstein manifold has parallel Ricci tensor, it is easy to observe that there do not exist the Einstein real hypersurfaces in a non-flat complex space form. For this Kon [10], studied and classified the pseudo-Einstein (that is there exist constants $\lambda, \mu$ such that the Ricci tensor $S$ satisfies $S=\lambda I+\mu \eta \otimes \eta$ ) real hypersurfaces of a complex space form $\bar{M}^{n}(c)$ when $n \geq 3$. Recently, Kim-Ryan [8] proved that every pseudo-Einstein hypersurface in $P_{2}(C)$ or $H_{2}(C)$ is the Hopf hypersurface. Now we recall some classification theorems of the pseudo-Einstein type real hypersurfaces in $P_{n}(C)$ (see $\left.[\mathbf{2}, \mathbf{1 0}]\right)$ or $H_{n}(C)$ (see [12]).

THEOREM B. Let $M^{2 n-1}(n \geq 3)$ be a real hypersurface of $P_{n}(C)$ with Fubini-study metric of constant holomorphic sectional curvature 4. Then $M$ is pseudo-Einstein if and only if $M$ is locally congruent to one of the following:

$\left(A_{1}\right)$ A geodesic hypersphere of radius $r$, where $0<r<\frac{\pi}{2}$.

$\left(A_{2}\right) A$ tube of radius $r$ over a totally geodesic $P_{k}(C)(1 \leq k \leq n-2)$, where $0<r<\frac{\pi}{2}$ and $\cot ^{2} r=\frac{k}{n-k-1}$.

(B) A tube of radius $r$ over a complex quadric $Q^{n-1}$ and $P_{n} R$, where $0<r<\frac{\pi}{4}$ and $\cot ^{2} 2 r=n-2$.

TheOREM C. Let $M^{2 n-1}(n \geq 3)$ be a real hypersurface of $H_{n}(C)$ with Bergman metric of constant holomorphic sectional curvature -4. Then $M$ is pseudo-Einstein if and only if $M$ is locally congruent to one of the following:

( $\left.A_{0}\right)$ A horosphere.

$\left(A_{1}\right)$ A geodesic hypersphere or a tube over a complex hyperbolic hyperplane $H_{n-1}(C)$.

Moreover, we remark that a tube over a totally geodesic $H_{l}(C)(1 \leq l \leq n-2)$ is known as a $A_{2}$-type hypersurface of $H_{n}(C), n \geq 3$. Note that real hypersurfaces of types $A_{1}$ and $A_{2}$ (without extra restriction $\cot ^{2} r=\frac{k}{n-k-1}$ ) in $P_{n}(C)$ and of types $A_{0}, A_{1}$ and $A_{2}$ in $H_{n}(C)$ are simply known as a real hypersurfaces of type $A$.

A Ricci soliton is a generalisation of Einstein metric and is defined on a Riemannian manifold $(M, g)$ by a vector field $V$ and a constant $\lambda$

$$
\left(£_{V} g\right)(X, Y)+2 S(X, Y)+2 \lambda g(X, Y)=0,
$$

where $£_{V}$ denotes the Lie-derivative operator along $V, S$ is the Ricci tensor of $g$ and $X, Y$ are arbitrary vector fields on $M$. It can be viewed as a fixed point of the Hamilton's Ricci flow: $\frac{\partial}{\partial t} g_{i j}=-2 R_{i j}$, as a dynamical system, on the space of Riemannian metrics modulo diffeomorphisms and scalings. For details we refer to Chow-Knopf [4]. Recently, Cho-Kimura [3] considered real hypersurfaces of a nonflat complex space form that admits the Ricci soliton with $V=\xi$ and proved that such hypersurface does not exist. For this reason, Cho-Kimura [3] defined the so-called $\eta$-Ricci soliton by taking $V=\xi$ and adding an extra term $\mu \eta \otimes \eta$ in the left-hand side 
of (2), i.e.

$$
\frac{1}{2} £_{\xi} g+S+\lambda g+\mu \eta \otimes \eta=0
$$

for constants $\lambda$ and $\mu$. Under this assumption they proved that $M$ is pseudo-Einstein (or $\eta$-umbilical). Moreover, as a generalisation of $\eta$-Ricci soliton, one may consider real hypersurfaces $M$ of a complex space form $\bar{M}^{n}(c)$ satisfying

$$
\frac{1}{2}\left(£_{\xi} g\right)(X, Y)+S(X, Y)+\lambda g(X, Y)=0,
$$

for all tangent vectors $X, Y$ orthogonal to $\xi$ and $\lambda$ is constant. We call this a generalised $\eta$-Ricci soliton. Note that there exist real hypersurfaces that admit a $\eta$-Ricci soliton and hence generalised $\eta$-Ricci soliton. In fact, it is straight forward to see that any $\eta$ umbilical real hypersurface of a complex space form admits such a structure. Thus, as a generalisation of Cho-Kimura's result we classify real hypersurfaces $M$ of complex space form $\bar{M}^{n}(c)$ satisfying equation (3). We discuss this matter in Section 4.

2. Real hypersurfaces in a complex space form. In this section we recall some basic equations and formulas that we shall use later on. For details about the real hypersurfaces of a complex space form we refer to Niebergall-Ryan [14]. Let $M$ be a real hypersurface of a Kaehler manifold $(\bar{M}, J, \bar{g})$. For any vector field $X$ tangent to $M$, we put

$$
\begin{aligned}
& J X=\varphi X+\eta(X) \xi \\
& J N=-\xi
\end{aligned}
$$

where $\varphi$ is a tensor field of type $(1,1), \eta$ is a 1 -form and $\xi$ is a unit vector field on $M$. We denote the induced metric of $M$ by $g$. From equation (4) it is easy to see that $(\varphi, \xi, \eta, g)$ gives an almost contact metric structure on $M$, that is

$$
\begin{gathered}
\varphi^{2} X=-X+\eta(X) \xi, \eta(\xi)=1, \\
g(\varphi X, \varphi Y)=g(X, Y)-\eta(X) \eta(Y),
\end{gathered}
$$

for all vector fields $X, Y$ on $M$. From these equations it is easy to see that $\varphi \xi=0$ and $\eta \circ \varphi=0$. The Gauss and Weingarten formulas for $M$ are given by

$$
\bar{\nabla}_{X} Y=\nabla_{X} Y+g(A X, Y), \bar{\nabla}_{X} N=-A X,
$$

where $\bar{\nabla}$ and $\nabla$ are the Levi-Civita connection of $\bar{M}$ and $M$, respectively. Making use of these formulas, equations (4) and (5) and $\bar{\nabla} J=0$ (as $\bar{M}$ is Kaehler) it follows that

$$
\begin{gathered}
\left(\nabla_{X} \varphi\right) Y=\eta(Y) A X-g(A X, Y), \\
\nabla_{X} \xi=\varphi A X
\end{gathered}
$$

where $A$ is the second fundamental tensor of $M$. Now we suppose that the Kaehler manifold $\bar{M}=\bar{M}(c)$ is a complex space form. Then we have the following Gauss and 
Codazzi equations:

$$
\begin{aligned}
R(X, Y) Z= & \frac{c}{4}\{g(Y, Z) X-g(X, Z) Y+g(\varphi Y, Z) \varphi X-g(\varphi X, Z) \varphi Y \\
& -2 g(\varphi X, Y) \varphi Z\}+g(A Y, Z) A X-g(A X, Z) A Y
\end{aligned}
$$

for any tangent vector fields $X, Y, Z$ on $M$. From equation (10), we get

$$
S X=\frac{c}{4}\{(2 n+1) X-3 \eta(X) \xi\}+h A X-A^{2} X,
$$

where $h$ is the trace of $A$. If the vector field $\xi$ is a principal curvature vector in a non-flat complex space form, i.e. $A \xi=\alpha \xi$, then $M$ is called the Hopf hypersurface of $\bar{M}(c)$. Such hypersurfaces have some remarkable properties. Note that for $c \neq 0, \alpha$ is constant (see $[6,10,11,14])$.

\section{Real hypersurfaces with semi parallel $T$.}

THEOREM 1. Let $M$ be real hypersurface of a non-flat complex space form. If the tensor $T$ is semi-parallel, then $M$ is locally congruent to a type A hypersurface.

Proof. By hypothesis, we have

$$
R(X, Y) T-T R(X, Y)=0
$$

from which we get

$$
g(R(X, Y) T Z, W)-g(R(X, Y) Z, T W)=0 .
$$

Setting $Z=W=\xi$ the foregoing equation yields

$$
g(R(X, Y) T \xi, \xi)=0 .
$$

Now, from equation (1), $T \xi=\varphi A \xi$ and hence equation (13) reduces to $g(R(X, Y) \varphi A \xi, \xi)=0$. Thus, in view of this we obtain from equation (10)

$$
\begin{aligned}
& \frac{c}{4}\{g(Y, \varphi A \xi) \eta(X)-g(X, \varphi A \xi) \eta(Y)\}+g(A Y, \varphi A \xi) g(A X, \xi) \\
& \quad-g(A X, \varphi A \xi) g(A Y, \xi)=0
\end{aligned}
$$

Next, putting $Y=\varphi A \xi$ and since $g(A \varphi A \xi, \xi)=0$, the foregoing equation implies that

$$
\frac{c}{4} g(\varphi A \xi, \varphi A \xi) \eta(X)+g(A \varphi A \xi, \varphi A \xi) g(A X, \xi)=0 .
$$

Finally, taking $X=\varphi A \varphi A \xi$ in equation (14) provides $g(A \varphi A \xi, \varphi A \xi)=0$. Making use of this in equation (14) and since $M$ is non-flat, we see that $\xi$ is principal, i.e. $A \xi=\alpha \xi$. Utilising this and taking $Y=Z=\xi$ in equation (12), we get $T R(X, \xi) \xi=0$. Let $X$ be any principal vector orthogonal to $\xi$ corresponding to the principal curvature $\lambda$, i.e. $A X=\lambda X$. Then $R(X, \xi) T \xi=0$ since $T \xi=0$. Also from the Gauss equation (10) it follows that $R(X, \xi) \xi=\left(\alpha \lambda+\frac{c}{4}\right) X$. Thus,

$$
0=T R(X, \xi) \xi=\left(\alpha \lambda+\frac{c}{4}\right)(A \varphi-\varphi A) X
$$


so that unless there is a principal curvature satisfying $\alpha \lambda+\frac{c}{4}=0$, we are finished by Theorem A. Suppose $\lambda$ is such a principal curvature so that $A X=\lambda X$ and $(A \varphi-$ $\varphi A) X \neq 0$. The well-known properties of principal curvatures of Hopf hypersurfaces (see [14, pp 245-246]) give a principal curvature $\mu$ such that $A \varphi X=\mu \varphi X$. Since $(A \varphi-\varphi A) X=(\mu-\lambda) X$ we have $\mu \neq \lambda$. This is a contradiction as the same argument applied to $\mu$ and $\varphi X$ gives $\alpha \mu+\frac{c}{4}=0$. This completes the proof.

REMARK 1. In [16], Pyo-Suh proved that a real hypersurface $M$ of a non-flat complex space form $\bar{M}^{n}(c), n \geq 2$, satisfying $£_{\xi} R=0$ is of type $A$. We can prove this result by applying Theorem 1 . In fact, Lie differentiating the identity

$$
g(R(X, Y) Z, W)+g(R(X, Y) W, Z)=0,
$$

using $£_{\xi} R=0$ and (1), it follows that $(R(X, Y) T) Z=0$.

Next we prove the following.

THEOREM 2. Let $M$ be real hypersurface of a non-flat complex space form with recurrent $T$. Then $M$ is locally congruent to one of type $A$ in $P_{n}(C)$ or $H_{n}(C)$.

Proof. By hypothesis $T$ is recurrent, i.e. there exists a 1 -form $\pi$ such that

$$
\left(\nabla_{X} T\right) Y=\pi(X) T Y,
$$

for all vector fields $Y, Z$ on $M$. Clearly $T$ is symmetric. Suppose $T$ has a non-zero eigenvalue $\sigma$, for otherwise $T=0$ and by Theorem A, $M$ will be congruent to one of type $A$ in $P_{n}(C)$ or $H_{n}(C)$. Let $Y$ be a unit vector and $T Y=\sigma Y$. Then by (15), we have

$$
\pi(X) g(T Y, Y)=g\left(\left(\nabla_{X} T\right) Y, Y\right)=g\left(\nabla_{X}(T Y), Y\right)-g\left(\nabla_{X} Y, T Y\right) .
$$

Using $T Y=\sigma Y$ the foregoing equation shows that

$$
(X \sigma) g(Y, Y)+\sigma g\left(\nabla_{X} Y, Y\right)-\sigma g\left(\nabla_{X} Y, Y\right)=\sigma \pi(X) g(Y, Y),
$$

which, in turn, gives $X \sigma=\sigma \pi(X)$. Writing this consequence as $d \sigma=\sigma \pi$ and operating this by $d$ (operator of exterior differentiation) and using the Poincaré lemma, $d^{2}=0$, we obtain

$$
0=d^{2} \sigma=d \sigma \wedge \pi+\sigma d \pi=\sigma(\pi \wedge \pi)+\sigma d \pi,
$$

i.e. $\sigma d \pi=0$. At this point we take an open set $\mathrm{N}$ of all points $p$ of $M$ such that $\sigma(p) \neq 0$. Then on $\mathrm{N}, d \pi=0$, i.e.

$$
\left(\nabla_{X} \pi\right) Z=\left(\nabla_{Z} \pi\right) X .
$$

Now, for any $X, Y$ and $Z \in T_{p} M$ and $p \in \mathrm{N}$, by differentiating (15) covariantly with respect to $Z$, we obtain

$$
\left(\nabla_{Z} \nabla_{X} T\right) Y=\left\{\left(\nabla_{Z} \pi\right) X\right\} T Y+\pi(Z) \pi(X) T Y .
$$

Interchanging $Z$ and $X$ we have

$$
\left(\nabla_{X} \nabla_{Z} T\right) Y=\left\{\left(\nabla_{X} \pi\right) Z\right\} T Y+\pi(X) \pi(Z) T Y .
$$


Making use of these equations, together with the Ricci identity and (16) we find that

$$
R(X, Z) T Y-T R(X, Z) Y=0 .
$$

Therefore, following the proof of Theorem 1 it is easy to see that $T=0$ and so $\sigma=0$ on $\mathrm{N}$. Thus, we arrive at a contradiction and hence $\varphi A=A \varphi$. Using Theorem A, we complete the proof.

\section{Generalised $\eta$-Ricci soliton.}

THEOREM 3. Let $M$ be real hypersurface of a non-flat complex space form admitting a generalised $\eta$-Ricci soliton. If the tensor $g(T X, Y)$ of $M$ vanishes for all $X, Y$ orthogonal to $\xi$, then $M$ is pseudo-Einstein.

Proof. In view of equation (1), the hypothesis $g(T X, Y)=0$, for all $X, Y$ orthogonal to $\xi$ implies $g((A \varphi-\varphi A) X, Y)=0$, for all $X, Y$ orthogonal to $\xi$, which is equivalent to

$$
\varphi A \varphi^{2} X-\varphi^{2} A \varphi X=0
$$

for all $X$ tangent to $M$. Operating this by $\varphi$ and replacing $X$ by $\varphi X$, the foregoing equation provides

$$
(A \varphi-\varphi A) X-g(A \varphi X, \xi) \xi+\eta(X) \varphi A \xi=0 .
$$

Since $M$ admits a generalised $\eta$-Ricci soliton, equation (3) is equivalent to

$$
g\left(\nabla_{\varphi X} \xi, \varphi Y\right)+g\left(\nabla_{\varphi Y} \xi, \varphi X\right)+2 S(\varphi X, \varphi Y)+2 \lambda g(\varphi X, \varphi Y)=0
$$

for all vectors $X, Y$ tangent to $M$. Making use of equations (9) and (11), the foregoing equation yields

$$
\varphi A \varphi^{2} X-\varphi^{2} A \varphi X+\varphi A^{2} \varphi X-h \varphi A \varphi X-\left\{2 \lambda+\frac{(2 n+1) c}{2}\right\} \varphi^{2} X=0
$$

for all vectors $X$ tangent to $M$. Therefore, use of (6) the last equation entails that

$$
\begin{gathered}
(A \varphi-\varphi A) X-g(A \varphi X, \xi) \xi+\eta(X) \varphi A \xi+\varphi A^{2} \varphi X \\
-h \varphi A \varphi X-\left\{2 \lambda+\frac{(2 n+1) c}{2}\right\} \varphi^{2} X=0 .
\end{gathered}
$$

Feeding equation (17) into (19) provides

$$
\varphi A^{2} \varphi X-h \varphi A \varphi X-\left\{2 \lambda+\frac{(2 n+1) c}{2}\right\} \varphi^{2} X=0 .
$$

Operating equation (20) by $\varphi$ we get an equation and replacing $X$ by $\varphi X$ in equation (20) gives another equation. Differentiating them yields

$$
\begin{aligned}
& \left(\varphi A^{2}-A^{2} \varphi\right) X+g\left(A^{2} \varphi X, \xi\right) \xi-\eta(X) \varphi A^{2} \xi \\
& \quad+h\{(A \varphi-\varphi A) X-g(A \varphi X, \xi) \xi+\eta(X) \varphi A \xi\}=0 .
\end{aligned}
$$


Thus, in view of equation (17), the preceding equation shows that

$$
\left(\varphi A^{2}-A^{2} \varphi\right) X+g\left(A^{2} \varphi X, \xi\right) \xi-\eta(X) \varphi A^{2} \xi=0 .
$$

In other words

$$
g\left(\left(\varphi A^{2}-A^{2} \varphi\right) X, Y\right)=0,
$$

for all tangent vectors $X, Y$ orthogonal to $\xi$. Now, operating equation (17) by $A$ gives

$$
\left(A^{2} \varphi-A \varphi A\right) X-g(A \varphi X, \xi) A \xi+\eta(X) A \varphi A \xi=0 .
$$

Further, replacing $X$ by $A X$, equation (17) transforms into

$$
\left(A \varphi A-\varphi A^{2}\right) X-g(A \varphi A X, \xi) \xi+g(A X, \xi) \varphi A \xi=0 .
$$

Adding equation (23) with (24) and taking into account equation (22) it follows that

$$
g(A X, \xi) g(\varphi A \xi, Y)+g(\varphi A \xi, X) g(A Y, \xi)=0,
$$

for all tangent vectors $X, Y$ orthogonal to $\xi$. Since $\varphi \xi=0$, the vector fields $\varphi^{2} A \xi$ and $\varphi A \xi$ are orthogonal to $\xi$. Therefore, if we replace $X$ by $\varphi^{2} A \xi$ and $Y$ by $\varphi A \xi$, then equation (25) shows $|\varphi A \xi|^{4}=0$, which implies $\varphi A \xi=0$, that is $A \xi=\alpha \xi$. This, together with the hypothesis $(g(A \varphi-\varphi A) X, Y)=0$, for all $X, Y$ orthogonal to $\xi$ implies that $A \varphi=\varphi A$. Moreover, using $A \xi=\alpha \xi$ in equation (11), we see that $S \xi=\beta \xi$, where $\beta=\frac{c(n-1)}{2}+h \alpha-\alpha^{2}$. Making use of equation (9), $\left.g(A \varphi-\varphi A) X, Y\right)=0$ in equation (18), we find that

$$
S(\varphi X, \varphi Y)+\lambda g(\varphi X, \varphi Y)=0 .
$$

Finally, replacing $X$ by $\varphi X$ and $Y$ by $\varphi Y$ in the foregoing equation and since $S \xi=\beta \xi$ we see that $M$ is pseudo-Einstein.

ACKNOWLEDGEMENTS. The author is very much grateful to the referee for reading the manuscript carefully and offering many valuable comments on it. In particular, the referee kindly suggested a shorter proof of Theorem 1 than its earlier version.

The author has been supported by the UGC (India) under the scheme Minor Research Project in Science, sanction no. F.PSW-106/09-10, dated 8 October 2009.

\section{REFERENCES}

1. J. Berndt, Real hypersurfaces with constant principal curvatures in complex hyperbolic space, J. Reine Angew. Math. 395 (1989), 132-141.

2. T. E. Cecil and P. J. Ryan, Focal sets and real hypersurfaces in complex projective space, Trans. Amer. Math. Soc. 269 (1982), 481-499.

3. J. T. Cho and M. Kimura, Ricci solitons and real hypersurfaces in a complex space form, Tohoku Math. J. 61(2) (2009), 205-212.

4. B. Chow and D. Knopf, The Ricci flow: An introduction, Mathematical Surveys and Monographs, Vol. 110 (American Mathematical Society, Providence, RI, 2004).

5. U-H. Ki, On real hypersurfaces with parallel Ricci tensor of a complex space form, Tsukuba J. Math. 13 (1989), 73-81.

6. U-H. Ki and Y. J. Suh, On real hypersurfaces of a complex space form, Math. J. Okayama Univ. 32 (1990), 207-221. 
7. U. K. Kim, Nonexistence of Ricci-parallel real hypersurfaces in $\mathrm{P}_{2} \mathrm{C}$ or $\mathrm{H}_{2} \mathrm{C}$, Bull. Korean Math. Soc. 41 (2004), 699-708.

8. H. S. Kim and P. J. Ryan, A classification of pseudo-Einstein hypersurfaces in $C P^{2}$, Diff. Geom. Appl. 26 (2008), 106-112.

9. M. Kimura, Real hypersurfaces and complex submanifolds in complex projective space, Trans. Amer. Math. Soc. 296 (1986), 137-149.

10. M. Kon, Pseudo-Einstein real hypersurfaces in complex space forms, J. Diff. Geom. 14 (1979), 339-354.

11. Y. Maeda, On real hypersurfaces of a complex projective space, J. Math. Soc. Japan 28 (1976), 529-540.

12. S. Montiel, Real hypersurfaces of a complex hyperbolic space, J. Math. Soc. Japan 37 (1985), 515-535.

13. S. Montiel and A. Romero, On some real hypersurfaces of a complex hyperbolic space, Geom. Dedicata 20 (1986), 245-261.

14. R. Niebergall and P. J. Ryan, Real hypersurfaces in complex space forms, in Tight and taut submanifolds (T. E. Cecil et al., Editors) (MSRI, Cambridge, UK, 1994), 233-305.

15. M. Okumura, On some real hyersurfaces of a complex projective space, Trans. Amer. Math. Soc. 212 (1975), 355-364.

16. Y.-S. Pyo and Y. J. Suh, Characterizations of real hypersurfaces in complex space forms in terms of curvature tensors, Tsukuba J. Math. 19 (1995), 163-172.

17. R. Takagi, On homogeneous real hypersurfaces of a complex projective space, Osaka J. Math. 10 (1973), 495-506.

18. R. Takagi, Real hypersurfaces in a complex projective space with constant principal curvatures I, II, J. Math. Soc. Japan, 27 (1975), 43-53, 507-516. 